Xavier, CC, Melo, SVA, Freitas, AFL, Contreira, AR \& Fiorese, L. (2020). Passion and athletic satisfaction in brazilian basketball college athletes. Research, Society and Development, 9(7): 1-21, e512974282.

\title{
Paixão e satisfação atlética em atletas brasileiras de basquetebol universitário
}

Passion and athletic satisfaction in brazilian basketball college athletes

Pasión y satisfacción atlética en las deportistas de baloncesto universitarias brasileñas

Recebido: 06/05/2020 | Revisado: 11/05/2020 | Aceito: 14/05/2020 | Publicado: 24/05/2020

\section{Caroline Carneiro Xavier}

ORCID: https://orcid.org/0000-0002-4469-3764

Universidade Estadual de Maringá, Brasil

E-mail: Carol.97.xavier@gmail.com

Sandro Victor Alves Melo

ORCID: https://orcid.org/0000-0002-2897-5999

Universidade Federal do Acre, Brasil

Universidade Estadual de Maringá, Brasil

E-mail:sandro.melo@ufac.br

Ana Flávia Lopes Freitas

ORCID: https://orcid.org/0000-0001-8995-254X

Universidade Estadual de Maringá, Brasil

E-mail: anaflavialfs@gmail.com

Andressa Ribeiro Contreira

ORCID: https://orcid.org/0000-0001-9331-3134

Universidade do Estado do Amazonas, Brasil

Universidade Estadual de Maringá, Brasil

E-mail: acontreira@uea.edu.br

Lenamar Fiorese

ORCID: https://orcid.org/0000-0003-1610-7534

Universidade Estadual de Maringá, Brasil

E-mail: lenamarfiorese@gmail.com 


\title{
Resumo
}

Paixão e satisfação atlética são variáveis da psicologia positiva relacionadas ao desempenho esportivo e investigadas no âmbito nacional e internacional. Objetivou-se analisar a paixão e satisfação atlética em atletas brasileiras de basquetebol universitário, buscando comparar a paixão e satisfação das atletas em função do tempo de experiência e desempenho e correlacionar as variáveis paixão e satisfação. Foram sujeitos 60 atletas, com média de idade 22,06 ( $\pm 3,01$ anos), experiência média de 10,13 ( $\pm 3,61$ anos), participantes dos Jogos Universitários Brasileiros de 2017. Os dados foram analisados pelos testes Wilcoxon, Friedman, U de Mann Whitney e coeficiente de correlação de Spearman ( $p<0,05)$. As atletas apresentaram predominância da paixão harmoniosa e mostraram-se mais satisfeitas nas dimensões treino e instrução $(M d=5,67)$. Ao comparar a paixão e satisfação atlética em função do tempo de experiência, as atletas mais experientes apresentaram-se mais apaixonadas harmoniosamente $(\mathrm{Md}=6,00)$ e mais satisfeitas nas dimensões contribuição da equipe para a tarefa e contribuição da equipe para o social $(\mathrm{Md}=5,33)(\mathrm{p}<0,05)$. Na comparação da paixão e satisfação atlética em função do ranking, foram observadas diferenças para as dimensões contribuição equipe para tarefa $(p=0,04)$ e contribuição equipe para o social $(\mathrm{p}=0,03)$, com resultados superiores para as atletas não medalhistas $(\mathrm{Md}=5,33)$. Foram observadas correlações entre a paixão em todas as dimensões da satisfação atlética $(\mathrm{p}>0,05)$, exceto para orçamento e diretoria médica $(\mathrm{p}<0,05)$. As atletas brasileiras de basquetebol universitário são apaixonadas harmoniosamente pela modalidade e percebem-se satisfeitas nos aspectos relacionados ao treino instrução fornecidos pelo treinador e quanto à sua própria contribuição com a equipe.

Palavras-chave: Paixão; Satisfação; Basquetebol.

\begin{abstract}
Passion and athletic satisfaction are variables of positive psychology studied in the sports context, related to performance, and investigated at the national and international levels. The objective of the study was to analyze the passion and athletic satisfaction in Brazilian college basketball athletes, seeking to compare the passion and satisfaction of the athletes according to the time of experience and performance and to correlate the variables passion and satisfaction. 60 athletes were subjects, with an average age of 22.06 ( \pm 3.01 years) average experience of 10.13 ( \pm 3.61 years), participants of the 2017 Brazilian University Games. The data were analyzed by Wilcoxon tests, Friedman, U of Mann Whitney and Spearman's correlation coefficient $(\mathrm{p}<0.05)$. The athletes showed a predominance of harmonious passion
\end{abstract}


and were more satisfied in the dimensions of training and instruction $(\mathrm{Md}=5.67)$. When comparing the passion and athletic satisfaction according to the time of experience, the more experienced athletes were more harmoniously passionate $(\mathrm{Md}=6.00)$ and more satisfied in the dimensions of the team's contribution to the task and the team's contribution to the social dimension $(\mathrm{Md}=5.33)(\mathrm{p}<0.05)$. In the comparison of passion and athletic satisfaction according to the ranking, differences were observed for the dimensions team contribution to task $(\mathrm{p}=0.04)$ and team contribution to social $(\mathrm{p}=0.03)$, with superior results for nonathletes medalists $(\mathrm{Md}=5.33)$. Correlations between passion were observed in all dimensions of athletic satisfaction $(\mathrm{p}<0.05)$, except for budget and medical management $(\mathrm{p}>0.05)$. Brazilian college basketball athletes are harmoniously passionate with the sport and find themselves satisfied in the aspects related to the training instruction provided by the coach and in their own contribution to the team.

Keywords: Passion; Satisfaction; Basketball.

\section{Resumen}

La pasión y la satisfacción atlética son variables positivas de psicología estudiadas en el contexto deportivo, relacionadas con el rendimiento e investigadas en nivel nacional e internacional. El objetivo del estudio fue analizar la pasión y la satisfacción atlética en las atletas de baloncesto universitarias brasileñas, buscando específicamente comparar la pasión y la satisfacción de estas deportistas según el tiempo de experiencia y rendimiento en la competencia y correlacionar las variables pasión y satisfacción. 60 atletas fueron sujetos, con edad media de 22.06 ( \pm 3.01 años) experiencia media de 10.13 ( \pm 3.61 años), participantes de los juegos universitarios brasileños de 2017. Para el análisis de datos se utilizaron las pruebas de wilcoxon, friedman, u de mann whitney y el coeficiente de correlación de spearman, adoptando $\mathrm{p}<0.05$. Las atletas mostraron un predominio de pasión armoniosa. En la satisfacción atlética, se verificó que las atletas estaban más satisfechas en las dimensiones de entrenamiento e instrucción (md = 5.67). Al comparar la pasión y la satisfacción atlética en función del tiempo de experiencia, las atletas expertas fueron más armoniosamente apasionadas $(m d=6.00)$ y más satisfechas en las dimensiones de la contribución del equipo a la tarea y la contribución del equipo a la dimensión social ( $\mathrm{md}=5,33)(\mathrm{p}<0,05)$. En la comparación de la pasión y la satisfacción atlética según el ranking, se observaron diferencias para las dimensiones contribución del equipo a la tarea $(\mathrm{p}=0.04)$ y contribución del equipo a lo social $(\mathrm{p}=0.03)$, con resultados superiores para las atletas no medallistas $(\mathrm{md}=5.33)$. Se observaron correlaciones entre la pasión y todas las dimensiones de la satisfacción atlética ( $\mathrm{p}$ 
$<0.05$ ), excepto el presupuesto y el manejo médico ( $\mathrm{p}>0.05$ ). Las deportistas de baloncesto universitarias brasileñas están armoniosamente enamorados del deporte y se sienten satisfechas con los aspectos relacionados con el entrenamiento proporcionado por el entrenador y con su propia contribución al equipo.

Palabras clave: Pasión; Satisfacción; Baloncesto.

\section{Introdução}

O estudo das variáveis psicológicas está diretamente ligado ao bem-estar e desempenho dos atletas. Nesse sentido, a verificação dos aspectos psicológicos dos atletas traz benefícios para a melhora da perfomance de equipes, ajudando na modulação de treinamentos e melhora nas escolhas de atletas durante situações decisivas no contexto esportivo de rendimento (Vieira, et al.,2010).

Diversas variáveis psicológicas vêm sendo estudadas no contexto esportivo como a motivação, estresse, autoconfiança, paixão, satisfação atlética, relação treinador atleta (Paludo \& Koller, 2010). A paixão, especificamente, é definida como uma forte inclinação para uma atividade que o indivíduo gosta, investe tempo e energia (Vallerand et al., 2006). Para o estudo dessa variável, os autores elaboraram o Modelo Dualístico da Paixão, caracterizando a paixão em dois componentes (paixão harmoniosa e a paixão obsessiva).

A paixão harmoniosa se caracteriza como não controladora, a sua internalização na identidade da pessoa é positiva e de livre escolha do praticante, conseguindo ajustar-se com outras atividades. Já na paixão obsessiva ocorre a internalização controlada na personalidade, gerando sentimentos negativos para a prática, pressões internas, tornando-se incompatível com outras atividades (Vallerand et al., 2006). Ressalta-se que ambos os tipos de paixão são relevantes para o desempenho dos atletas, no entanto é necessário o equilíbrio entre os componentes para evitar os efeitos estressores advindos do contexto esportivo e do excesso de treinamento.

Segundo a literatura, a paixão está associada a outras variáveis psicológicas como motivação (Ferreira, 2013); felicidade (Sousa, 2013); bem-estar e sacrifício (Cid \& Louro, 2010), sendo extensivamente investigada no contexto internacional. Dentre os estudos realizados encontra-se o desenvolvido por Cid e Louro (2010), que investigou se a prática de natação é uma paixão ou sacrifício, com o objetivo principal de analisar a relação existente 
entre os tipos de paixão. Os atletas apresentaram uma paixão harmoniosa pela modalidade gerando assim resultados positivos em relação ao seu desempenho e suas habilidades.

Sousa (2013) abordou a paixão pelo surf e o impacto na motivação e felicidade dos praticantes, os quais obtiveram níveis elevados para a paixão harmoniosa e para a obsessiva, caracterizando o meio aquático como imprevisível, gerando assim pressões ao praticar a modalidade, mas caracterizando a paixão harmoniosa como amenizadora dos sentimentos negativos. Já a investigação de Teixeira (2011) verificou a influência do tipo de paixão na orientação motivacional dos atletas de futebol e futsal, identificando que os atletas possuem uma paixão harmoniosa pela modalidade, relacionando-se com a tarefa.

Outra variável psicológica relevante para a permanência e persistência dos atletas no contexto do rendimento é a satisfação atlética. Segundo Chelladurai e Riemer (1997) a satisfação atlética caracteriza-se como um estado afetivo positivo originado de uma avaliação complexa das estruturas, dos processos e dos resultados associados com a experiência esportiva. Nesse sentido, a satisfação é observada pela discrepância dos desejos do atleta e a percepção dos domínios psicológicos, físicos e do ambiente externo.

A satisfação do atleta não depende somente de aspectos pessoais, mas também de estruturas do ambiente de treinamento, equipe médica, treinador,companheiros de equipe (Chelladurai \& Riemer, 1997). Estudos ligados à satisfação atlética têm observado suas associações com outras variáveis psicológicas como motivação (Mizoguchi, Balbim, \& Vieira, 2013); perfeccionismo (Balbim, Nascimento Junior, \& Vieira, 2013); liderança (Chelladurai \& Riemer, 1997), em diferentes modalidades esportivas como futsal, beisebol, rúgbi e esporte escolar.

Dentre os estudos nacionais abordados encontra-se o desenvolvido por Balbim et al. (2013), que analisou o nível de satisfação e perfeccionismo de atletas profissionais de futsal do norte do Paraná; Mizoguchi et al.(2013) que investigaram o estilo parental, motivação e satisfação de atletas de beisebol, enquanto que a pesquisa de Rodrigues e Saldanha (2016) analisou a liderança e satisfação no esporte escolar.

Ao considerar as variáveis apresentadas, observa-se a ausência de estudos realizados no contexto do basquetebol. Este esporte, segundo Tavares (2002), possui um caráter dinâmico em que cada segundo de jogo é decisivo para o resultado final de uma partida, assim atletas mais apaixonados e satisfeitos pela modalidade podem ser mais capazes de se ajustar às 
situações de jogo para obter desfechos positivos. Dessa forma, avaliar estas variáveis pode acarretar benefícios aos treinadores, comissões técnicas e atletas, contribuindo para o aumento do desempenho e bem-estar no contexto esportivo, o que justifica a relevância do presente estudo.

Além disso, destaca-se a necessidade de verificação da paixão e da satisfação em relação à modalidade basquetebol feminino, bem como no âmbito do desporto universitário, o qual possui pouca divulgação, visibilidade e incentivo. Isso porque acredita-se que atletas mais apaixonadas por sua modalidade podem apresentar melhores tomadas de decisões, demonstram-se mais autoconfiantes e permanecem mais tempo em sua modalidade. Ainda, o sentimento de satisfação em relação a equipe, treinador, desempenho para tarefa e pessoal tornam as atletas mais acessíveis para instruções e comandos dados pelo seus treinadores obtendo resultados positivos e a potencialização de suas habilidades (Vallerand et al., 2007; Borrego et al., 2010).

Outros aspectos que demonstram a relevância da pesquisa referem-se à recente validação da Escala da Paixão para o contexto esportivo nacional por Prates (2015), a qual possibilita a realização de pesquisas no contexto esportivo nacional. Diante da importância dos aspectos apresentados, este estudo objetivou analisar a paixão e satisfação em atletas de basquetebol universitário, buscando especificamente: 1) comparar a paixão e satisfação das atletas em função do tempo de experiência e desempenho na competição; 2) Correlacionar a paixão e satisfação das atletas de basquetebol universitário.

\section{Metodologia}

O estudo caracteriza-se como descritivo-correlacional do tipo transversal (Thomas, Nelson, \& Silverman, 2012). Segundo Thomas et al. (2012), a pesquisa descritiva objetiva descrever o perfil de determinada população estabelecendo as relações entre variáveis.

Fizeram parte do campeonato 75 atletas de 8 equipes femininas representando os estados do Mato Grosso - MT, Ceará - CE, Paraná - PR, Rio Grande do Sul - RS, - DF, Goiás - GO, Amazonas - AM, Bahia - BA e Distrito Federal - DF. Destes estados apenas a coleta do representante do Amazonas não aconteceu em função da dificuldade logística do setor de hospedagem. Dessa forma, participaram do estudo 60 atletas brasileiras de basquetebol universitário, com média de idade 22,06 ( $\pm 3,01$ anos), com tempo médio de 
experiência 10,13 ( $\pm 3,61$ anos), de 7 equipes de diferentes estados do Brasil, classificadas para o $65^{\circ}$ Jogos Universitários Brasileiros (JUBS) da segunda divisão, no ano de 2017, realizado na cidade de Goiânia no estado de Goiás.

Para avaliar a paixão das atletas foi utilizada a Escala da Paixão (PS) desenvolvida originalmente por Vallerand e Houlfort (2003) e validada para o contexto esportivo brasileiro por Prates (2015). A escala é constituída por 14 itens, respondidos numa escala de 7 pontos, em que 1 corresponde a "não concordo em nada" e 7 corresponde a "concordo muito fortemente". A escala é dividida em 3 dimensões: Paixão Obsessiva; Paixão Harmoniosa e Critério da paixão.

Para avaliação do nível de satisfação atlética foi utilizado o questionário Athlete Satisfaction Questionnaire (ASQ) criado por Chelladurai e Riemer (1997) e validado por Borrego et al. (2010) que realizaram a versão portuguesa, que se constitui por 14 dimensões contendo 53 itens no total. Nestas 14 dimensões compreendem desempenho individual, prestação da equipe, utilização da habilidade, estratégia, tratamento pessoal, treino e instrução, contribuição da equipa para a tarefa, contribuição da equipe para o social, ética, integração da equipe, dedicação pessoal, orçamento, pessoal médico e agentes externos. O instrumento é respondido numa escala tipo likert de 7 pontos, em que 1 corresponde a nada satisfeito e 7 corresponde a extremamente satisfeito.

A coleta foi realizada pela aprovação do Comitê Permanente de Ética em Pesquisas Envolvendo Seres Humanos (COPEP) da Universidade Estadual de Maringá (parecer 1.324.411/2015). Para aplicação dos questionários durante o período de competição, primeiramente foi solicitado a autorização da CBDU (Confederação Brasileira de Desportos Universitários).

O próximo passo foi estabelecer contato com cada técnico, capitã ou responsável da equipe para o consentimento do convite as atletas. Os dados foram coletados uma hora antes de cada partida no local da competição. As atletas receberam um Termo de Consentimento Livre e Esclarecido para a participação do estudo aprovado pelo Comitê Permanente de Ética (COPEP) da Universidade Estadual de Maringá, juntamente uma ficha de identificação contendo idade, tempo de prática, nível competitivo e tempo de prática com o treinador e os questionários de paixão (PS) e satisfação (QSA) os instrumentos foram entregues, explicados e respondidos de forma individualizada, com duração média de 18 minutos. 
Os dados foram analisados no pacote estatístico SPSS versão 20.0. Para verificação da normalidade dos dados foi utilizado o teste Kolmogorov Smirnov (n>50), obtendo-se distribuição não normal. Assim, os resultados foram apresentados por meio de estatística descritiva (Mediana e Intervalo Interquartílico) e estatística inferencial. Os testes utilizados foram Friedman, U de Man Whitney e coeficiente de correlação de Sperman, adotando-se $\mathrm{p}<0,05$ em todas as análises.

\section{Resultados e Discussão}

Ao identificar a paixão das atletas brasileiras de basquete universitário verificou-se predominância da paixão harmoniosa $(\mathrm{Md}=5,80)$ sobre a paixão obsessiva $(\mathrm{Md}=3,20)$ com diferenças significativas $(\mathrm{p}=0,001)$. A paixão harmoniosa pode ser entendida, segundo Vallerand et al. (2003), como uma integração positiva e autônoma da atividade em relação à personalidade, permitindo que o atleta participe de forma efetiva, amenizando os efeitos negativos dessa atividade, acarretando benefícios e sentimentos positivos ao praticá-la. 
Tabela 1. Paixão e satisfação das atletas de basquete feminino universitário ( $n=60)$.

\begin{tabular}{|c|c|c|}
\hline Variáveis & Mediana & Quartis \\
\hline Paixão & (Md) & (Q1-Q3) \\
\hline 1 Paixão Obsessiva & 3,20 & $2,25-4,20$ \\
\hline 2 Paixão Harmoniosa & 5,80 & $5,05-6,35$ \\
\hline Satisfação atlética & (Md) & (Q1-Q3) \\
\hline 1 Desempenho individual & 5,00 & $4,00-5,67$ \\
\hline 2 Desempenho de equipe & 5,00 & $4,33-6,00$ \\
\hline 3 Utilização de habilidades & 5,33 & $4,67-5,83$ \\
\hline 4 Estratégias & 5,33 & $4,00-5,83$ \\
\hline 5 Tratamento pessoal & 5,20 & $4,40-6,15$ \\
\hline 6 Treino instrução & 5,67 & $4,41-6,33$ \\
\hline 7 Contribuição tarefa & 5,16 & $4,41-6,00$ \\
\hline 8 Contribuição social & 5,33 & $4,33-6,00$ \\
\hline 9 Ética & 5,33 & $4,08-6,33$ \\
\hline 10 Integração equipe & 4,75 & $4,25-5,93$ \\
\hline 11 Dedicação pessoal & 5,50 & $4,56-6,00$ \\
\hline 12 Orçamento & 4,67 & $3,33-5,67$ \\
\hline 13 Diretoria médica & 4,00 & $2,50-5,50$ \\
\hline 14 Agentes externos & 3,75 & $2,56-4,68$ \\
\hline
\end{tabular}

Diferenças estatisticamente e significativas para $\mathrm{p}<0,05$ entre: Satisfação atlética: a) 1 e 14 ; b) 2 e 13 ; c) 2 e 14; d) 3 e 13 ; e) 3 e 14; f) 4 e 14; g) 5 e 13 ; h) 5 e 14; i) 6 e 12; j) 7 e 13 ; k) 8 e 13; 1) 8 e 14 ; m) 9 e 13 ; n) 9 e 14 ; p) 10 e 14 ; q) 11 e 14.

O estudo realizado por Cid e Louro (2010) apresentou resultados semelhantes em relação aos valores elevados para a paixão harmoniosa $(\mathrm{Md}=5,40)$ em praticantes de natação, indicando que os atletas desempenham tal modalidade pelo gosto que possuem por esta atividade e não por pressões internas, obtendo um efeito protetor aos efeitos negativos causados pelo esporte. Estes aspectos também foram observados na pesquisa de Schellenberg 
(2011) no voleibol universitário, em que a paixão harmoniosa obteve valores superiores $(\mathrm{Md}=5,58)$. A partir destes resultados o autor caracteriza a paixão harmoniosa ligada a situações em que o atleta percorreu durante sua formação com autonomia, acarretando benefícios à internalização em sua personalidade.

Resultados contrários aos do presente estudo foram identificados por Ferreira (2013) com praticantes de surf e bodyboard de ambos os sexos. Os autores observaram valores elevados para os dois tipos de paixão (paixão harmoniosa e paixão obsessiva), justificados devido ao estresse gerado pelos desafios que são encontrados na prática em meio aquático. Assim, a paixão harmoniosa é vista como fator amenizador dos efeitos negativos e a paixão obsessiva caracterizada pelos desafios gerados pela prática, diferentemente das modalidades que são praticadas em ambientes previsíveis.

Em relação à satisfação atlética (Tabela 1), as atletas mostraram-se mais satisfeitas nas dimensões treino e instrução com o valor mais expressivo $(\mathrm{Md}=5,67)$, seguido por dedicação pessoal $(\mathrm{Md}=5,50)$, utilização de habilidades, estratégias, contribuição social e ética $(\mathrm{Md}=5,33)$. Foram observados valores inferiores para as dimensões agentes externos $(\mathrm{Md}=3,75)$, diretoria médica $(\mathrm{Md}=4,00)$ e orçamento $(\mathrm{Md}=4,67)$.

O estudo de Alves (2010) com atletas juvenis de basquetebol corrobora os achados desta pesquisa ao identificar a satisfação dos atletas nas dimensões treino instrução, tratamento pessoal, desempenho individual e desempenho da equipe. Os autores descrevem esses resultados articulando a importância do treinador em relação à formação do atleta, tendo em vista que a satisfação dos atletas é dependente das instruções transmitidas pelo treinador, empregando as habilidades, selecionando e utilizando estratégias para o direcionamento de sua equipe, além da relevância do tratamento pessoal dado aos atletas.

Para Balbim et al. (2013), na pesquisa com atletas profissionais de futsal masculino foram encontrados valores significativos para a dimensão desempenho da equipe que apresentou menor valor em relação às demais, os atletas também apresentaram valores elevados para dimensões relacionadas ao seu desempenho e em relação ao treino e instrução, os quais vão ao encontro desta pesquisa. Os autores relacionam este resultado pelo baixo valor apresentado em relação ao perfeccionismo dos atletas, os quais se caracterizavam por serem organizados e comprometidos em relação à sua equipe, treinamento e objetivos. 
Em relação à satisfação dos atletas de futsal masculino adulto, Nascimento Junior, Vieira, Souza e Vieira (2011) identificaram valores semelhantes para a dimensão treino e instrução aos das atletas de basquetebol universitário, enfatizando novamente a importância da presença do treinador para o sentimento de satisfação e resultados positivos da equipe, concluindo ainda que fatores extrínsecos como o estilo parental de suporte emocional dos responsáveis contribuem para bons níveis de satisfação atlética.

Ao comparar as dimensões da paixão das atletas brasileiras de basquetebol universitário em função do tempo de experiência esportiva não foram encontradas diferenças significativas $(p>0,05)$ (Tabela 2). No entanto, foi possível observar que, para ambos os grupos a paixão harmoniosa foi superior à paixão obsessiva.

Para Vallerand et al., (2003), a paixão harmoniosa ameniza os sentimentos negativos gerado pelas situações promovidas pela experiência esportiva negativa, mantendo o atleta na prática da atividade que gosta por mais tempo, buscando assim, a excelência de suas habilidades técnicas e táticas. Ademais, a paixão harmoniosa contribui para que o resultado possa ser sempre positivo e desprovido de obrigações e sentimentos negativos, tornando o atleta cada vez mais experiente em suas tomadas de decisões. 
Tabela 2. Comparação da paixão e satisfação atlética em função do tempo de experiência das atletas brasileiras de basquete universitário.

\begin{tabular}{|c|c|c|c|}
\hline Variáveis & $\begin{array}{c}\text { Até } 9 \text { anos } \\
\quad(n=27)\end{array}$ & $\begin{array}{l}\text { Acima } 10 \text { anos } \\
\qquad(n=33) \\
\text { Md (Q1-Q3) }\end{array}$ & $\mathbf{p}$ \\
\hline Paixão Obsessiva & $3,60(2,80-4,40)$ & $3,00(1,80-4,20)$ & 0,079 \\
\hline Paixão Harmoniosa & $5,80(5,00-6,20)$ & $6,00(5,40-6,50)$ & 0,344 \\
\hline Desempenho individual & $5,00(4,33-5,67)$ & $5,00(4,00-6,00)$ & 0,928 \\
\hline Desempenho de equipe & $4,67(3,33-5,67)$ & $5,33(4,67-6,00)$ & 0,118 \\
\hline Utilização de habilidades & $5,17(4,17-5,50)$ & $5,50(4,91-5,50)$ & 0,129 \\
\hline Estratégias & $5,00(4,00-5,83)$ & $5,50(4,08-5,83)$ & 0,666 \\
\hline Tratamento pessoal & $5,20(4,00-6,00)$ & $5,20(4,60-6,33)$ & 0,387 \\
\hline Treino instrução & $5,67(4,67-3,33)$ & $5,67(4,33-6,67)$ & 0,742 \\
\hline Contribuição tarefa & $5,00(4,00-5,67)$ & $5,33(4,67-6,33)$ & $\mathbf{0 , 0 2 8} *$ \\
\hline Contribuição social & $4,67(3,67-6,00)$ & $5,33(4,83-6,33)$ & $0,025 *$ \\
\hline Ética & $5,33(3,67-6,00)$ & $5,67(4,83-6,33)$ & 0,068 \\
\hline Integração equipe & $4,75(3,25-6,00)$ & $4,75(4,25-4,75)$ & 0,451 \\
\hline Dedicação pessoal & $5,50(5,00-5,75)$ & $5,50(4,50-6,25)$ & 0,596 \\
\hline Orçamento & $4,67(3,33-5,67)$ & $4,67(3,16-5,83)$ & 0,994 \\
\hline Diretoria médica & $4,00(2,25-5,50)$ & $4,00(2,50-5,25)$ & 0,677 \\
\hline Agentes externos & $3,50(2,25-5,50)$ & $3,75(3,00-4,50)$ & 0,512 \\
\hline
\end{tabular}

*Nível de significância $p<0,05$.

Quanto à comparação das dimensões da satisfação atlética em função do tempo de experiência esportiva das atletas brasileiras de basquetebol universitário, foram encontradas diferenças estatisticamente significativas entre os grupos $(\mathrm{p}<0,05)$ para as dimensões Contribuição da equipe para a tarefa $(\mathrm{Md}=5,33)$ e Contribuição da equipe para o social $(\mathrm{Md}=5,33)$. 
A satisfação atlética é moderada por fatores importantes como o tempo de treino e competição (Borrego et al., 2010). Assim, atletas mais experientes possuem maiores vivências em âmbito competitivo e de treinamento e são mais propensos a níveis elevados de satisfação. Resultados semelhantes foram encontrados no estudo de Rosado, Mesquita, Correia, e Colaço (2009), em que a satisfação dos atletas seniores foi superior aos de atletas juvenis para a dimensão desempenho individual $(\mathrm{Md}=5,09)$, caracterizando os atletas mais experientes como mais satisfeitos em relação a maioria das dimensões.

Na pesquisa desenvolvida Passos et al. (2015), objetivou-se comparar a satisfação atlética em jogadores de futsal em função do nível de experiência destes atletas. Os achados foram contrários aos desta pesquisa, revelando diferenças significativas para as dimensões integração de equipe e orçamento, que foram mais elevadas para atletas com menor tempo de experiência esportiva. Tais resultados podem decorrer do fato de que atletas mais experientes podem mostrar-se menos satisfeitos com as situações de dificuldade encontradas na equipe e falta de investimentos/recursos financeiros.

Na Tabela 3 são apresentados os resultados da comparação da paixão e satisfação atlética das atletas de basquete feminino universitário em função do desempenho na competição (medalhistas e não medalhistas). Para a paixão, os resultados demonstraram que as atletas brasileiras de basquete universitário não apresentaram diferenças ( $>$ >0,05) em função do desempenho, tanto para a paixão harmoniosa quanto para a paixão obsessiva.

No estudo desenvolvido por Bonneville-Roussy, Lavigne, e Vallerand (2011), foi realizada a comparação entre o tipo de paixão e desempenho de músicos profissionais e estudantes de música. Os resultados foram contrários aos desta pesquisa, pois foram observados valores significativos para a paixão harmoniosa para os estudantes de música, que apresentaram menor desempenho em relação aos músicos profissionais. 
Tabela 3. Comparação da paixão e satisfação atlética em função do desempenho das atletas brasileiras de basquete universitário.

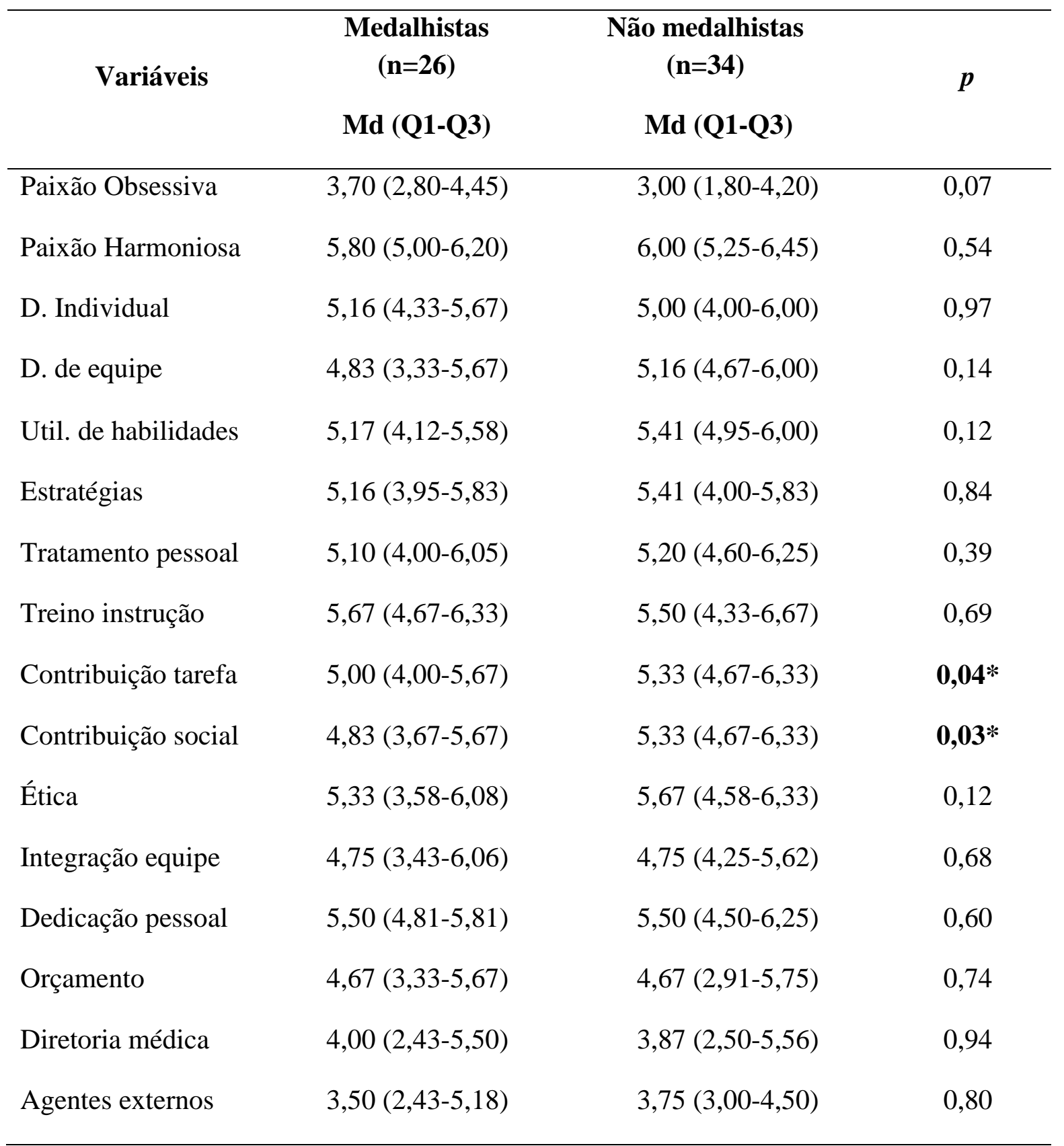

Nota: D. Individual= Desempenho individual; D. de equipe $=$ Desempenho de equipe; Util. de habilidades $=$ Utilização de habilidades. *Nível de significância $\mathrm{p}<0,05$.

Ao comparar as dimensões da satisfação em função do desempenho (Tabela 3) as análises demonstraram diferenças estatisticamente significativas para as dimensões contribuição tarefa $(p=0,04)$ e contribuição da equipe para o social $(p=0,03)$, com resultados superiores sendo apresentados para as atletas não medalhistas $(\mathrm{Md}=5,33)$. No estudo 
(CC BY 4.0) | ISSN 2525-3409 | DOI: http://dx.doi.org/10.33448/rsd-v9i7.4282

apresentado por Gomes, Pereira, e Pinheiro (2009) em jogadores de futebol de categorias femininas e masculinas de vários escalões os resultados mostraram-se distintos, tendo valores significativos de satisfação no desempenho individual. Dessa forma, o autor identificou atletas mais satisfeitos em relação ao seu desempenho individual, tendo como resultado final maior coesão de grupo, aplicação das habilidades de forma correta em cada situação e melhor desempenho coletivo.

Ao analisar a correlação entre a paixão e satisfação atlética, verificou-se correlação significativa positiva entre paixão harmoniosa e a maioria das dimensões da satisfação atlética $(p<0,05)$, exceto para orçamento e diretoria médica $(p>0,05)$. As correlações identificadas foram de fracas a moderadas, com valores entre $0,26<\mathrm{r}<0,56$, indicando correlações mais elevadas para contribuição social, utilização de habilidades, dedicação pessoal e desempenho individual. Conforme os resultados observados, verifica-se que à medida em que se eleva a paixão harmoniosa das atletas pelo seu esporte, o nível de satisfação se eleva na mesma proporção. 
Tabela 4. Correlação entre Paixão e satisfação atlética das atletas de basquete feminino universitário.

\begin{tabular}{ccc}
\hline Variáveis & Paixão Obsessiva & Paixão Harmoniosa \\
\hline Desempenho individual & 0,00 & $\mathbf{0 , 4 0}$ \\
Desempenho de equipe & 0,20 & $\mathbf{0 , 3 5}$ \\
Utilização de habilidades & 0,24 & $\mathbf{0 , 5 5 *}$ \\
Estratégias & 0,01 & $\mathbf{0 , 2 7} *$ \\
Tratamento pessoal & $-0,00$ & $\mathbf{0 , 2 6} *$ \\
Treino instrução & $-0,02$ & $\mathbf{0 , 2 6 *}$ \\
Contribuição tarefa & 0,01 & $\mathbf{0 , 2 8 *}$ \\
Contribuição social & 0,15 & $\mathbf{0 , 5 9 *}$ \\
Ética & 0,18 & $\mathbf{0 , 3 6} *$ \\
Integração equipe & 0,16 & $\mathbf{0 , 3 7 *}$ \\
Dedicação pessoal & 0,08 & $\mathbf{0 , 5 0 *}$ \\
Orçamento & 0,02 & 0,25 \\
Diretoria médica & $-0,13$ & 0,12 \\
Aggentes externos & 0,23 & $\mathbf{0 , 3 2} *$ \\
\hline
\end{tabular}

*Nível de significância $\mathrm{p}<0,05$.

Estes resultados podem ser compreendidos a partir do Modelo Dualístico da Paixão que busca analisar os sentimentos que se manifestam em diferentes momentos na experiência esportiva, propondo que as pessoas se envolvem em várias atividades ao longo de sua vida com a finalidade de satisfazer suas necessidades psicológicas básicas (Vallerand et al., 2003). Nessa perspectiva, entende-se que a paixão harmoniosa se relaciona à internalização autônoma da modalidade sobre a personalidade do atleta, de modo que a prática esportiva promove sensações positivas diante dos aspectos físicos e psicológicos presentes no cotidiano esportivo (Vieira, Mizoguchi, Contreira, Passos, \& Costa, 2015). Tais aspectos podem se estender às experiências e relações estabelecidas nesse contexto, como as capacidades adquiridas, 
desempenho, relacionamento, autonomia para a tarefa, estado de fluxo e satisfação (Vieira et al., 2015).

Assim, entende-se que atletas apaixonadas harmoniosamente pelo seu esporte tendem a sentir-se satisfeitas por meio de situações que são atribuídas através de resultados e atribuições sociais resultantes da observação daqueles que pertencem ao contexto esportivo, ou seja, essas atribuições são o encontro entre a harmonia do que é vivenciado pelo atleta e suas exigências (Borrego et al., 2010).

\section{Considerações Finais}

O objetivo do estudo foi analisar a paixão e satisfação atlética em atletas brasileiras de basquetebol universitário. No geral, observou-se que as atletas se apresentaram apaixonadas harmoniosamente pela sua modalidade, com níveis de satisfação elevados para as dimensões treino instrução, estratégias e utilização de habilidades e ética.

Verificou-se que as atletas com maior tempo de experiência no esporte apresentaramse mais apaixonadas harmoniosamente em relação às atletas menos experientes. As atletas mais experientes apresentaram-se mais satisfeitas nas dimensões contribuição para tarefa e contribuição para o social. Além disso, observou-se que quanto mais apaixonadas harmoniosamente pelo seu esporte, mais satisfeitas as atletas se sentem com sua experiência esportiva, exceto quando considerado o orçamento e diretoria médica.

A avaliação dos aspectos psicológicos como a paixão e satisfação atlética no contexto do basquetebol feminino universitário contribui para a identificação de fatores que podem contribuir para a inserção e permanência das atletas nesse contexto de pouco incentivo e divulgação, além de corroborar para os aspectos de bem-estar e melhora do desempenho, o que se considera fundamental para treinadores, psicólogos do esporte e professores de educação física, em busca da criação de ambientes esportivos harmoniosos para as jovens atletas.

Para futuros estudos sugere-se a avaliação de outras variáveis da psicologia positiva nesta população como as necessidades psicológicas básicas, esperança e relacionamento treinador-atleta, com vistas a compreender o perfil de atletas e fatores intervenientes na prática do basquetebol feminino. Além disso, o estudo destas variáveis contribui para conhecimentos relevantes para a preparação completa das atletas no contexto esportivo de rendimento. 


\section{Referências}

Alves, I. (2010). Relação entre a Coesão, o Clima Motivacional e a Satisfação em Modalidades Colectivas. (2007), 2007.

Balbim, G. M., Nascimento Junior, J. R. A. do, \& Vieira, L. F. (2013). Análise do nível de satisfação e perfeccionismo de atletas profissionais de futsal do Estado do Paraná. Conexões, 11(2), 15-30. https://doi.org/10.20396/conex.v11i2.8637615.

Bonneville-Roussy, A., Lavigne, G. L., \& Vallerand, R. J. (2011). When passion leads to excellence: The case of musicians. Psychology of Music, 39(1), 123-138. https://doi.org/10.1177/0305735609352441.

Borrego, C. M. C., Leitão, J. C., Alves, J., Silva, C., \& Palmi, J. (2010). Análise confirmatória do Questionário de Satisfação do Atleta: versão Portuguesa. Psicologia: Reflexão e Crítica, 23(1), 110-120. https://doi.org/10.1590/s0102-79722010000100014.

Chelladurai, P., \& Riemer, H. A. (1997). A Classification of Facets of Athlete Satisfaction. Journal of Sport Management, 11(2), 133-159. https://doi.org/10.1123/jsm.11.2.133.

Cid, L., \& Louro, H. (2010). Praticar Natação É Uma Paixão Ou Um Sacrifício? Estudo Da Relação Entre O Tipo De Paixão Que O Atleta Sente Pela Modalidade E a Sua Orientação Motivacional. Revista de Iberoamericana de Psicología de Ejercicio y el Deporte, 5(1), 99114.

Ferreira, J. S. M. (2013). Paixão e Orientação Motivacional no Surf e no Bodyboard. Estudo da Relação entre o Tipo de Paixão, os Objetivos de Realização e o Rendimento dos Atletas. 141.

Gomes, A. R., Pereira, A. P., \& Pinheiro, A. R. (2009). Liderança, coesão e satisfação em equipas desportivas: um estudo com atletas Portugueses de futebol e futsal. Psicologia: Reflexão e Crítica, 21(3), 482-491. https://doi.org/10.1590/s0102-79722008000300017. 
Leite, M. A. F. J., Sasaki, J. E., Zanetti, H. R., Santos, S. T., Abrahão, C. A. F., Chaves, A. D., \& Mendes, E. L. (2015). Nível de coesão grupal e satisfação de atletas de rugby. Coleção Pesquisa em Educação Física, 14(3), 125-132.

Mizoguchi, M. V., Balbim, G. M., \& Vieira, L. F. (2013). Estilo parental, motivação e satisfação de atletas de beisebol: Um estudo correlacional. Revista da Educacao Fisica, 24(2), 215-223. https://doi.org/10.4025/reveducfis.v24.2.16282.

Nascimento Junior, J. R. A. do, Vieira, L. F., Souza, E. A. de, \& Vieira, J. L. L. (2011). Nível de satisfação do atleta e coesão de grupo em equipes de futsal adulto. Revista Brasileira de Cineantropometria e Desempenho Humano, 13(2), 138-144. https://doi.org/10.5007/19800037.2011v13n2p138.

Paludo, S. dos S., \& Koller, S. H. (2010). Psicologia Positiva: uma nova abordagem para antigas questões. Paidéia (Ribeirão Preto), 17(36), 9-20. https://doi.org/10.1590/s0103$863 \times 2007000100002$.

Passos, P. C. B., Costa, L. C. A. da, Belem, I. C., Contreira, A., Vieira, L. F., \& Both, J. (2015). A satisfação do atleta de futsal e o tempo de experiência no esporte. Revista Brasileira de Ciência e Movimento, 23(3), 64-69.

Prates, M. E. F. (2015). A paixão pela atividade docente de professores universitários de educação física. Universidade Estadual de Maringá.

Rodrigues, V. de M., \& Saldanha, A. A. W. (2016). Liderança e Satisfação no Esporte Escolar: Teste da Hipótese da Congruência do Modelo Multidimensional de Liderança. Psicologia: Ciência e Profissão, 36(3), 653-667. https://doi.org/10.1590/19823703001492014.

Rosado, A., Mesquita, I., Correia, A., \& Colaço, C. (2009). Relação entre esgotamento e satisfação em jovens praticantes desportivos. Rev. Port. Cien. Desp., 9(2), 56-67.

Rubio, K. (2000). Psicologia do Esporte - Interfaces, Pesquisa e Intervenção (1 ${ }^{\mathrm{a}}$ ). Brasil. 
Schellenberg, B. J. I. (2011). Passion and Coping: Relationships With Burnout and Goal Attainment In Collegiate. Phys. Rev. E, (June), 53.

Sousa, C. J. M. de. (2013). A Paixão pelo Surf e o Impacto na Motivação e Felicidade dos Praticantes. https://doi.org/10.1016/j.copsyc.2014.12.004.

Tavares, F. (2002). Análise da estrutura e dinâmica do jogo nos jogos desportivos. In V. J. Barbanti, A. C. Amadio, J. O. Bento, \& A. T. Marques (Orgs.), Esporte e Atividade Física Interação Entre Rendimento e Saúde (1º, p. 368). Brasil.

Teixeira, P. (2011). Paixão no Desporto - Orientação Motivacional e Tipo de Paixã o em jovens Atletas federados em futebol de 11 e futsal.

Thomas, J. R., Nelson, J. K., \& Silverman, S. J. (2012). Métodos de pesquisa em atividade física $\left(6^{\mathrm{a}} ;\right.$ R. D. de S. Petersen, Org.). Porto Alegre.

Vallerand, R., \& Houlfort, N. (2003). Passion at work: Toward a new conceptualization. In S. W. Gilliland, D. D. Steiner, \& D. P. Skarlickid (Orgs.), Emerging Perspectives on Values in Organizations, Greenwich (p. 175-204). Greenwich.

Vallerand, R. J., Mageau, G. A., Ratelle, C., Léonard, M., Blanchard, C., Koestner, R., ... Marsolais, J. (2003). Les Passions de 1'Âme: On Obsessive and Harmonious Passion. Journal of Personality and Social Psychology, 85(4), 756-767. https://doi.org/10.1037/00223514.85.4.756.

Vallerand, R. J., Rousseau, F. L., Grouzet, F. M. E., Dumais, A., Grenier, S., \& Blanchard, C. M. (2006). Passion in sport: A look at determinants and affective experiences. Journal of Sport \& Exercise Psychology.

Vallerand, R. J., Salvy, S. J., Mageau, G. A., Elliot, A. J., Denis, P. L., Grouzet, F. M. E., \& Blanchard, C. (2007). On the role of passion in performance. Journal of Personality, 75(3), 505-534. https://doi.org/10.1111/j.1467-6494.2007.00447.x. 
Vieira, L. F., Mizoguchi, M. V., Contreira, A. R., Passos, P. C. B., \& Costa, L. C. A. da. (2015). Modelo dualistico da paixão: Um olhar no processo de aderência ao esporte. In J. V. do Nascimento, E. R. de Souza, V. Ramos, \& J. C. S. Rocha (Orgs.), Educação Física e Esporte: Convergindo para novos caminhos... (1º ed, p. 513-530). Frorianópolis: UDESC.

Vieira, L. F., Vissoci, J. R. N., de Oliveira, L. P., \& Vieira, J. L. L. (2010). Psicologia do esporte: Uma área emergente da psicologia. Psicologia em Estudo, 15(2), 391-399. https://doi.org/10.1590/S1413-73722010000200018.

\section{Porcentagem de contribuição de cada autor no manuscrito}

Caroline Carneiro Xavier autor centralizado - 20\%

Sandro Victor Alves Melo autor centralizado - 20\%

Ana Flávia Lopes Freiras da Silva autor centralizado - 20\%

Andressa Ribeiro Contreira autor centralizado - 20\%

Lenamar Fiorese autor centralizado - 20\% 